\title{
Essential functions linked with structural disorder in organisms of minimal genome
}

Rita Pancsa ${ }^{1}$ and Peter Tompa ${ }^{1,2^{*}}$

\begin{abstract}
Intrinsically disordered regions (IDRs) of proteins fulfill important regulatory roles in most organisms. However, the proteins of certain endosymbiont and intracellular pathogenic bacteria with extremely reduced genomes contain disproportionately small amounts of IDRs, consisting almost entirely of folded domains. As their genomes co-evolving with their hosts have been reduced in unrelated lineages, the proteomes of these bacteria represent independently evolved minimal protein sets. We systematically analyzed structural disorder in a representative set of such minimal organisms to see which types of functionally relevant longer IDRs are invariably retained in them. We found that a few characteristic functions are consistently linked with conformational disorder: ribosomal proteins, key components of the protein production machinery, a central coordinator of DNA metabolism and certain housekeeping chaperones seem to strictly rely on structural disorder even in genome-reduced organisms. We propose that these functions correspond to the most essential and probably also the most ancient ones fulfilled by structural disorder in cellular organisms.
\end{abstract}

Reviewers: This article was reviewed by Michael Gromiha, Zoltan Gaspari and Sandor Pongor.

Keywords: Intrinsically disordered, Structural disorder, Genome reduction, Endosymbiont, Minimal genome, Disorder prediction, Essential function, Genome-reduced bacterium, Chaperone function

Abbreviations: ATP, Adenosine triphosphate; GTP, Guanosine triphosphate; Hsp70, Seat shock protein 70; IDP, Intrinsically disordered protein; IDR, Intrinsically disordered region; LDR, Long disordered region; NMR, Nuclear magnetic resonance; PDB, Protein Data Bank; PIR, Protein information resource; SSB, Single stranded DNA binding protein; ssDNA, Single stranded DNA

\section{Findings}

Minimal bacteria lack structurally disordered regions Intrinsically disordered proteins (IDPs) and regions (IDRs) of proteins function as ensembles of unfolded conformations [1-4]. They play important regulatory and signaling roles [5], and take central positions in cellular interaction networks [6, 7] as specialists of protein-protein [8-10], protein-RNA [11] as well as protein-DNA interactions [12]. Due to the diverse functional advantages of structural disorder, IDPs/IDRs are widely employed for diverse functional purposes in all three kingdoms of life [13] as well as in viruses [14].

\footnotetext{
* Correspondence: ptompa@vub.ac.be

${ }^{1}$ Structural Biology Research Center (SBRC), Flanders Institute for

Biotechnology (VIB), Vrije Universiteit Brussel (VUB), 1050 Pleinlaan 2, Brussels, Belgium

${ }^{2}$ Institute of Enzymology, Research Centre for Natural Sciences, Hungarian Academy of Sciences, 1117 Budapest, Magyar Tudósok körútja 2., Budapest, Hungary
}

(c) 2016 The Author(s). Open Access This article is distributed under the terms of the Creative Commons Attribution 4.0 International License (http://creativecommons.org/licenses/by/4.0/), which permits unrestricted use, distribution, and reproduction in any medium, provided you give appropriate credit to the original author(s) and the source, provide a link to the Creative Commons license, and indicate if changes were made. The Creative Commons Public Domain Dedication waiver (http://creativecommons.org/publicdomain/zero/1.0/) applies to the data made available in this article, unless otherwise stated. tions $[13,15]$ that IDRs are more abundant in eukaryotes than prokaryotes, and highlighted that their abundance not only depends on the complexity of the organisms but also on their lifestyle [16]. Upon analyzing conformational disorder in complete proteomes, it became apparent that in certain endosymbiotic and intercellular pathogenic bacteria that have undergone extreme genome reduction, structural disorder is almost completely lacking [16]. Endosymbiotic bacteria live exclusively within their host, mostly inside specific, dedicated insect cells [17], thus static environmental conditions and a steady supply of nutrients are granted by their host. As they did not need to adapt to environmental changes for hundreds of millions of years [18], their gene sets have been cut to the acceptable minimum [19] by losing entire regulatory and signaling pathways as well and a radical erosion of their remaining proteins, especially at the expense of their IDRs [20]. They have 
also often undergone functional convergence with other co-resident symbiotic bacteria resulting in metabolic complementarity and co-dependency $[18,19,21]$. Although they have incredibly reduced genomes, they still differ from organelles since they retain relatively robust gene sets that are considered complete enough to enable autonomous life under nutrient-rich, intracellular conditions. Furthermore, their genes are not commonly transferred to the host genome, which is common in the case of organelles [19]. Interestingly, in some of these organisms, high mutation rates coupled with genetic drift makes their proteins susceptible to misfolding, demanding the extensive assistance of chaperones [19]. As these minimal organisms have undergone independent paths of genome reduction in their respective hosts [19], and belong to different phylogenetic clades, their proteomes can be considered as independently evolved minimal protein sets, restricted to proteins of universal importance or of specific function required for the symbiosis [19]. Therefore, with the identification and systematic analysis of IDRs that have been consistently preserved within minimal bacteria, here we uncover and describe the most essential cellular functions relying on structural disorder.

\section{Identifying long disordered regions within \\ minimal proteomes}

We obtained the representative bacterial complete proteomes (<15\% co-membership threshold) from the PIR database (release 2015-04, [22]) and selected the ones with extreme genome reduction $(<580$ proteins; this threshold warrants that a few Mycoplasma species are included, but they do not overly dominate the data, and that the obtained proteomes are all well-annotated). Luckily, the resulting 13 proteomes represented diverse phylogenetic clades (Table 1).

Structural disorder was predicted for all the associated proteins by IUPred [23, 24], which is a conservative disorder prediction method showing good correspondence [25] with the consensus disorder patterns of MobiDB [26]. The average fraction of disordered residues in the obtained proteomes ranged between 1 and $12 \%$, with a median of $3.7 \%$ that is extremely low compared to other organisms [16]. Residues with an IUPred score $>0.5$ were considered as disordered and all stretches of at least 20 consecutive disordered residues were identified, which will be hitherto referred to as long disordered regions (LDRs). The number of identified LDRs ranged between 1 and 57 including ribosomal proteins, but only 0 to 41 excluding ribosomal proteins (with medians of 17 and 7 regions, respectively).

\section{Functions relying on structural disorder in minimal proteomes}

Ribosomal proteins contained a large fraction of the detected LDRs (See Additional file 1: Table S1). The L2,
L4, L15, and L34 protein components of the large ribosomal subunit and S12 and S13 of the small subunit contained LDRs in more than half of the investigated 13 species, while many other subunits also contained LDRs in multiple species of different clades. This is of no surprise, as ribosomal proteins are known to universally rely on structural disorder [27] that enables the formation of tightly packed ribonucleoprotein complexes with ribosomal RNAs.

Table 1 presents all the non-ribosomal proteins (by gene names) that have retained LDRs in at least three minimal proteomes belonging to at least two different bigger prokaryotic clades. The list includes components of the transcription (DNA-dependent RNA polymerase subunits beta and beta' and sigma factor /rpoB, $-\mathrm{C}$ and $-\mathrm{D} /$ ) and translation (translation initiation factor $2 / \mathrm{infB} /$, peptide chain release factor $1 / \mathrm{prfA} /$ ) machineries, two chaperones (the $60 \mathrm{kDa}$ GroEL /groL/ chaperonin and DnaK), single-stranded DNA-binding protein /ssb/, and the transmembrane ATP-dependent zinc metalloprotease FtsH.

For these 9 proteins, all the retained homologs were aligned by Custal Omega 1.2.2. [28] and the corresponding disorder predictions were projected onto the alignments to see the positional conservation of LDRs.

RNA polymerase subunits (beta, beta' and sigma, rpoB, rpoC and rpoD, in Table 1) gave the weakest signal. Although they are annotated in all species, they show no sign of disorder in most of the minimal organisms and reference bacteria. For the beta subunit, the few detected LDRs are located internally, while for the beta', they mainly occur at the C-terminal. The sigma factor has a highly disordered internal loop in two minimal organisms belonging to Gammaproteobacteria, just like in E. coli (see Protein Data Bank (PDB; [29]) ID: 4YG2 [30]), and also in Mycoplasma hominis. Nonetheless, we have failed to find any evidence in the literature for the disordered nature of any particular region in these subunits.

Translation initiation factor 2 (IF2, infB in Table 1) is a GTPase essential for binding initiator transfer RNA to the $30 \mathrm{~S}$ ribosomal subunit and recruiting the $50 \mathrm{~S}$ subunit to the initiation complex [31]. The identified homologs show very large deviations in length and disorder pattern within the N-terminal region preceding the GTP-binding domain. Although in the three reference bacteria IF2 has a long, largely disordered N-terminus, the corresponding LDR has only been retained in three minimal organisms. This N-terminal flexible region promotes the joining of the two subunits [32] and makes IF2 largely extended in the complex (see PDB IDs: 3J4J, 3JCN and 3JCJ [33]).

In protein chain release factor 1 (prfA in Table 1), the C-terminal half consistently displays predicted disorder values that fluctuate around the order-disorder threshold. Even though in most minimal organisms this region 
Table 1 Non-ribosomal proteins that retained LDRs in at least three minimal organisms of at least two different bacterial clades

\begin{tabular}{|c|c|c|c|c|c|c|c|c|c|c|c|}
\hline Taxon ID & Clade & Species & rроB & rpoC & rpoD & $\operatorname{infB}$ & prfA & groL & dnak & ssb & $\mathrm{ftsH}$ \\
\hline 1053648 (115) & $\mathrm{BPB}$ & Cand. Tremblaya princeps PCVAL & $\checkmark$ & $\checkmark$ & ND & $\varnothing$ & $\varnothing$ & $\checkmark$ & $\checkmark$ & $\varnothing$ & $\varnothing$ \\
\hline 1343077 (137) & $\mathrm{BPB}$ & Cand. Nasuia deltocephalinicola str. NAS-ALF & ND & ND & ND & $\varnothing$ & ND & ND & $\checkmark$ & $\checkmark$ & $\varnothing$ \\
\hline 573234 (169) & APB & Hodgkinia cicadicola (strain Dsem) & ND & ND & ND & ND & ND & ND & $\checkmark$ & $\varnothing$ & $\varnothing$ \\
\hline 1266371 (175) & $\mathrm{BPB}$ & Cand. Tremblaya phenacola PAVE & $\checkmark$ & ND & ND & ND & $\checkmark$ & $\checkmark$ & $\checkmark$ & ND & $\varnothing$ \\
\hline $667013(206)$ & GPB & Cand. Carsonella ruddii DC & ND & ND & ND & ND & ND & ND & ND & $\varnothing$ & $\varnothing$ \\
\hline 871271 (206) & BPB & Zinderia insecticola (strain CARI) & ND & ND & ND & ND & ND & ND & ND & $\checkmark$ & $\varnothing$ \\
\hline $1415657(\mathbf{2 4 3})$ & BAC & endosymbiont of Llaveia axin axin & ND & ND & ND & $\checkmark$ & ND & ND & $\checkmark$ & $\varnothing$ & $\checkmark$ \\
\hline 1206109 (273) & GPB & Cand. Portiera aleyrodidarum BT-B-HRs & ND & ND & ND & $\checkmark$ & ND & $\checkmark$ & $\checkmark$ & $\varnothing$ & ND \\
\hline 482235 (448) & FIR & Phytoplasma mali (strain AT) & ND & ND & ND & ND & ND & ND & $\checkmark$ & ND & $\checkmark$ \\
\hline 347256 (529) & TEN & Mycoplasma hominis (strain ATCC 23114) & $\checkmark$ & $\checkmark$ & $\checkmark$ & ND & ND & $\varnothing$ & $\checkmark$ & $\checkmark$ & $\checkmark$ \\
\hline $515618(540)$ & GPB & Riesia pediculicola (strain USDA) & ND & ND & $\checkmark$ & ND & ND & $\checkmark$ & $\checkmark$ & $\checkmark$ & ND \\
\hline 107806 (572) & GPB & Buchnera aphidicola subsp. A. pisum (strain APS) & ND & $\checkmark$ & $\checkmark$ & $\checkmark$ & $\checkmark$ & $\checkmark$ & $\checkmark$ & $\checkmark$ & ND \\
\hline 1318617 (572) & TEN & Cand. Mycoplasma girerdii & ND & ND & ND & ND & $\checkmark$ & $\varnothing$ & $\checkmark$ & $\checkmark$ & $\checkmark$ \\
\hline $83333(4306)$ & GPB & Escherichia coli strain K12 & ND & ND & $\checkmark$ & $\checkmark$ & $\checkmark$ & ND & $\checkmark$ & $\checkmark$ & $\checkmark$ \\
\hline $435590(3982)$ & BAC & Bacteroides vulgatus strain ATCC 8482 & ND & ND & ND & $\checkmark$ & ND & ND & $\checkmark$ & $\checkmark$ & $\checkmark$ \\
\hline $224308(4197)$ & FIR & Bacillus subtilis strain 168 & $\checkmark$ & $\checkmark$ & ND & $\checkmark$ & $\checkmark$ & $\checkmark$ & $\checkmark$ & $\checkmark$ & ND \\
\hline
\end{tabular}

Taxon ID: the taxon identifier of the organisms with the number of their proteins annotated by UniProt in brackets. Clade: an abbreviation of the corresponding phylogenetic clade (APB Alphaproteobacteria, BPB Betaproteobacteria, GPB Gammaproteobacteria, BAC Bacteroidetes, FIR Firmicutes, TEN Tenericutes). Species and strain information is followed by columns showing information on the 9 proteins with retained LDRs. Different marks mean that i) the given protein was not annotated in the given organism (missing or does not show recognizable sequence homology ( $\varnothing$ )); ii) the protein is not disordered (ND), meaning that the given protein was annotated, but it does not contain an LDR in the given species, and iii) the protein has at least one LDR ( $\checkmark$ ). Data for non-minimal reference bacteria of three different clades are indicated in the last three lines in bold. A similar table with all corresponding UniProt identifiers indicated in the appropriate cells is available as Additional file 1: Table S2

does not fulfil the criteria of LDRs, the observed tendency is in good agreement with the large domain movements that are required for its interaction with the ribosome and accurate translation termination (see PDB IDs: 1RQ0, 4V7P, 5J4D and 1ZBT [34, 35]).

Interestingly, chaperones seem to rely on structural disorder the most. For example, the C-terminal tail of GroEL preserved its disordered nature in most minimal and reference species (Fig. 1a). Although the E. coli sequence is not predicted as disordered, this is certainly a misprediction because the respective region is missing from available X-ray structures (Fig. 1a). The highly flexible, hydrophilic tails protrude into the cavity of the ball-shaped chaperonin cage formed by GroEL/GroES subunits and assist the correct folding of proteins [36]. Multiple studies collectively confirmed that truncation of the tail impairs the efficient refolding of substrate proteins [36-39], however its specific molecular role in the chaperonin reaction cycle is still under debate $[38,39]$.

The C-terminal tail of chaperone protein DnaK (the Hsp70 homolog of bacteria) is the best preserved nonribosomal LDR in the investigated minimal and reference organisms (Fig. 1b). The tail region enhances chaperone activity and cellular survival upon stress in DnaK [40] and mediates interactions with co-chaperones containing a tetratricopeptide repeat domain in certain Hsp70 proteins $[41,42]$.
Single stranded DNA binding protein (SSB) is a universally conserved protein that binds ssDNA in a sequence-independent manner, and forms oligomers [43] while sliding on ssDNA [44-46]. The $>50$ residues long, disordered tails of the monomers remain disordered in the complex [47] and play essential hub roles in DNA replication, recombination and repair by orchestrating the action of an array of genome maintenance proteins $[44,46,48,49]$. Interestingly, the last nine amino acids provide the docking site for all the known partners $[46,50]$, while the amino acid composition and length of the conserved LDR (Fig. 1c) is thought to affect ssDNA binding mode preferences [51].

Finally, FtsH is an ATP-dependent zinc metallopeptidase that resides in the membrane and plays an essential role in the quality control of integral membrane proteins [52-54]. It is present in the 7 largest of the selected minimal organisms, with differentially positioned LDRs in four of them. The best-preserved disordered segment resides in its cytoplasmic AAA domain (see PDB IDs: 2R62 and 1IXZ), most probably conferring flexibility on the domain, but there are also compositionally biased LDRs in the $\mathrm{N}$ - and $\mathrm{C}$-terminal regions of the protein.

Most of the identified LDRs are genuine disordered regions that fulfill their functions as conformational ensembles and thus are missing from available structures of homologous proteins (see PDB structures: RpoD—4YG2, 


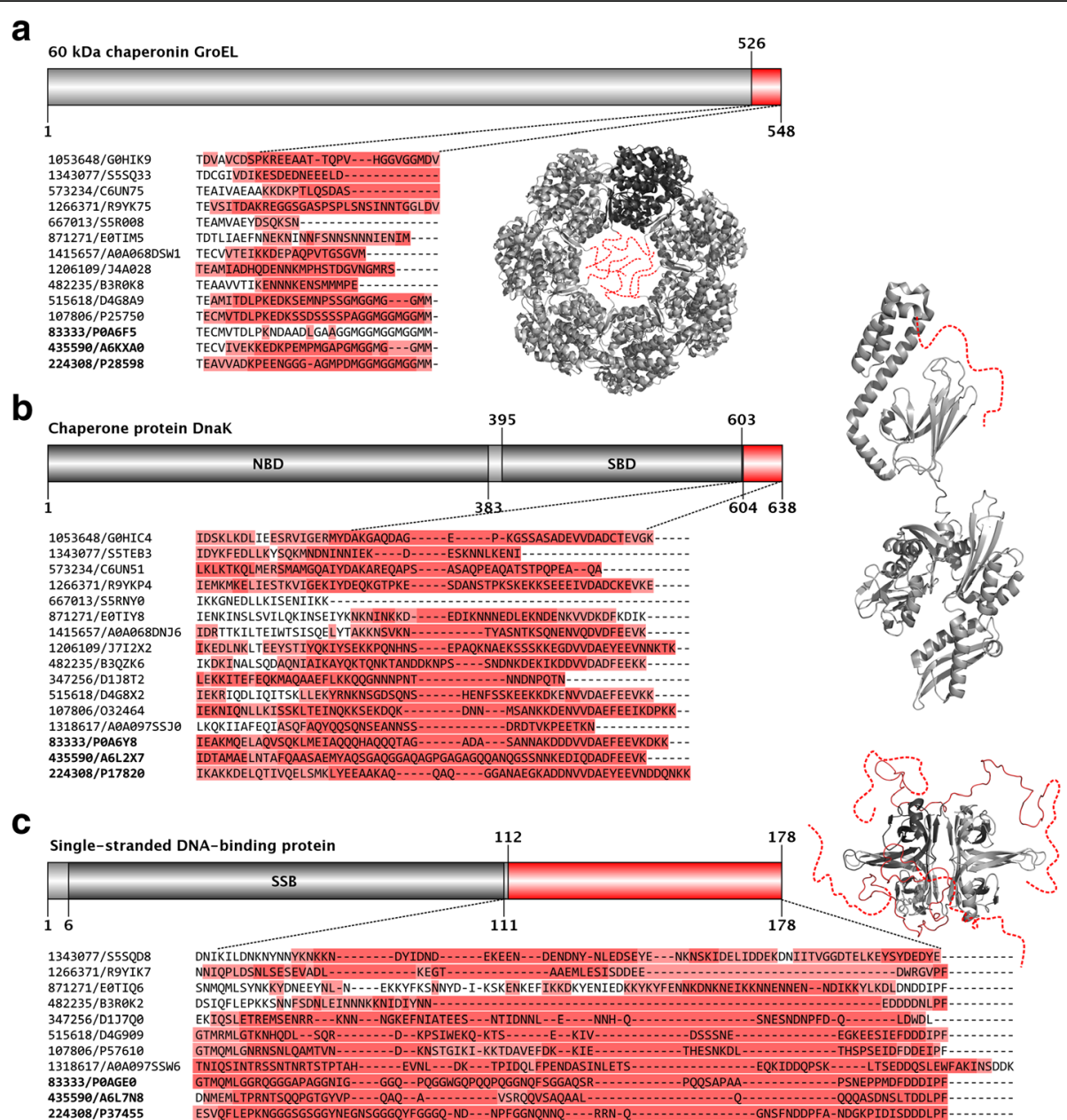

Fig. 1 Conserved disorder in chaperones and hub proteins of minimal organisms. Domain maps, structures, sequence and disorder conservation are depicted for the three proteins (GroEL (a) DnaK (b) and SSB (c)) with conserved long disordered regions. On the grey domain maps, the residue boundaries of conserved disordered segments (in red) and known domains (in darker grey) are provided. The red regions of the domain maps complemented by a few residue positions around are also highlighted as Clustal Omega 1.2.2. multiple sequence alignments below the domain maps. The sequences of the minimal and reference organisms are identified by their Taxonomy/UniProt identifiers, and are depicted in the same order as in Table 1. In the alignments the background of the residues are colored according to the corresponding IUPred predictions; residues with a score $>0.5$ in darker red, while residues with a score between 0.5 and 0.4 in lighter red. The structures of the corresponding E. coli proteins (PDB: 2NWC for GroEL, 2KHO for DnaK and 1QVC for SSB) are also depicted in light grey with the conserved disordered segments marked by red or added as red dashed lines. In the heptameric GroEL and tetrameric SSB structures one chain is depicted by darker grey than the others

InfB-3JCN, PrfA-1ZBT, GroEL-2NWC, DnaK-2$\mathrm{KHO}, \mathrm{SSB}-1 \mathrm{QVC})$. When repeating the analysis by requiring a minimum of 30 consecutive residues for an LDR, we could still identify 7 ribosomal proteins (Additional file 1: Table S3) and 4 other proteins (Additional file 1: Table S4; RpoD, InfB, DnaK, and $\mathrm{SSB})$ that retained LDRs in at least three minimal organisms from at least two clades.

We have also repeated the whole analysis with the ESpritz X-ray prediction method [55] using its conservative version ( $5 \%$ false positive prediction rate). The observed tendencies were largely preserved. Out of the 18 ribosomal proteins displaying conserved LDRs by IUPred, three did not show conserved LDRs with ESpritz, while another four only fulfilled the criteria with ESpritz (Additional file 2: Table S5). Regarding other proteins, with the exception of PrfA, all identified proteins listed in Table 1 displayed conserved LDRs also by ESpritz in the same regions as predicted by IUPred, while the replicative DNA helicase /dnaB/, protein GrpE /grpE/ and ribonuclease 3 /rnc/ were newly identified (Additional file 2: Table S6). We have checked the IUPred predictions of these novel proteins and confirm that they have just missed the criteria for conserved LDRs by displaying LDRs in one or two minimal organisms. When requiring a minimum of 30 
consecutive residues for an LDR, 7 ribosomal proteins (Additional file 2: Table S7) and 5 other proteins were retained, namely RpoC, InfB, DnaK, SSB and GrpE (Additional file 2: Table S8).

\section{Discussion}

The positional conservation of certain LDRs within minimal organisms of different bacterial lineages implies that structural disorder is essential for certain functions in the cell. It has always been thought to be essential in the functioning of the ribosome, not only because many ribosomal proteins contain disordered segments [27], but also because it facilitates the critical domain movements of important translation initiation and termination factors. Structural disorder has also been implicated in the functioning of RNA- and protein chaperones [56], and chaperones have been suggested to be one of the most disordered functional protein class in the proteome. Besides classical chaperones [36, 40], fully disordered stress proteins also can have chaperone activity [57], and thus the observation that two chaperones and other proteins playing a role in protein homeostasis ( $\mathrm{GrpE}$ and $\mathrm{FtsH}$ ) have retained their high level of structural disorder in organisms of minimal genome, fits strongly with this trend.

DNA/RNA-binding proteins are also among functional classes that abound in structural disorder, with disorder contributing to DNA binding, RNA binding and proteinprotein interactions, such as is transcription factors $[11,58]$. In full agreement, we found a conspicuous enrichment for structural disorder in transcription/ translation-related proteins in organisms of reduced genome. In addition, structural disorder is also central to proteins of hub function, due to structural adaptability accompanying induced folding, enabling IDPs to mediate multiple interactions; in addition, short interaction motifs enable very high functional density of IDRs [6]. This is manifested in the structural disorder of the C-terminal tail of SSB, probably the most universally conserved disordered hub that mediates specific interactions with different binding partners through a single short linear interaction motif [48], thereby orchestrating the different steps of DNA processing.

In all, the general functional feature of IDPs/IDRs that emerges from our study is that the most essential functions in which long IDRs are indispensable are the ones in which they mediate or regulate interactions with other macromolecular partners, e.g. RNA or proteins. In these, specific recognition has to be combined with the lack of strict sequence or surface characteristics. Typical examples are chaperones, in which IDRs contribute to recognizing ill-defined misfolded states of unrelated proteins, RNA-binding proteins in which IDRs bind distinct RNAs of variable secondary and tertiary structural features, and DNA-binding proteins, in which disordered regions are either directly involved in contacting DNA [58] or mediate domain movements involved in recognizing ssDNA, irrespective of its sequence. The advantage provided by structural adaptability of IDRs in these functions is unparalleled, and thus we conclude that these functions are amongst the most essential and most ancient functional roles fulfilled by structurally disordered regions in cellular organisms.

\section{Reviewers' comments \\ Reviewer 1: Michael Gromiha \\ Reviewer comments:}

In this work, the authors systematically analyzed structural disorder in a set of minimal organisms and showed that few characteristic functions are linked with conformational disorder. Further, they suggested that these functions correspond to the most essential ones fulfilled by structural disorder in cellular organisms. The analysis has been carried out extensively with specific examples to RNA polymerase, chaperone protein, single stranded DNA binding proteins, DnaK and so on. The work is interesting and the data provide new insights.

The following comments may be addressed for improvements.

1. The results obtained with negative dataset may be discussed.

Authors' response: We thank the reviewer for appreciating our work and his suggestions for improvements. We are not entirely sure what the reviewer means by negative dataset. Since the investigated proteomes are extremely minimalized, which affect both the number and the length of proteins, in some of them even the otherwise essential proteins listed in Table 1 are missing. Also, in many of them the disordered regions either disappeared or shortened to an extent that they do not fulfil our criteria of LDRs (Fig. 1 indicates that even though in some cases the disordered regions are shorter, they are mostly preserved). Now we added orthologs from reference bacteria of different phylogenetic groups to address if the identified regions are generally disordered or only due to the minimalistic nature of the investigated species. We find that many are also disordered in the reference proteomes. Now we also validate our results by using another method for disorder prediction.

2. The threshold value of 580 proteins for selecting the proteins with extreme genome reduction may be justified.

Authors' response: We have chosen the threshold of 580 for proteome size because above this threshold the obtained proteome set would have been too much biased towards Mycoplasma species. With this threshold we ensured that only two Mycoplasmas are selected, but obligate endosymbionts of different phylogenetic groups are still well represented. Also, somewhat above this 
threshold there are non-Mycoplasma proteomes in which the proteins were not annotated, only numbered and hence they could not have been used for this analysis.

\section{Reviewer 2: Zoltan Gaspari}

\section{Reviewer comments:}

This paper describes some important findings about the role of intrinsic protein disorder in minimal genomes. Its original and can be of interest for researchers working in the respective field. Although I think that the work contains novel findings, the volume of the data processed and the novel information provided is a bit limited. I think that the amount of sequences analyzed makes a more detailed study possible and I make some recommendations for this below that the authors might consider to improve the manuscript.

- The authors used one prediction algorithm (IUPred), one threshold (0.5) for classifying residues and one (20 aa) for identifying long regions. Where such necessarily subjective choices should be made, it can be important to prove the robustness of the main conclusions by repeating the analysis with some parameters varied. Can the authors provide such considerations?

Authors' response: We thank the reviewer for appreciating our work and his suggestions for improvements. IUPred is a very widely used and trusted method that shows good correspondence with consensus predictions obtained based on many different methods. In many of the previous analyses we and others have repeated the predictions with different methods and the identified tendencies were always preserved. This is why we thought one trusted method should be enough to point out such tendencies. Also, some of the methods that can be locally used on complete proteomes, for example VSL2B is known to predict very similar patterns but with elevated absolute values, meaning that it usually overestimates the number and extent of disordered regions compared to consensus disorder patterns. In this analysis overprediction is definitely not desired because we wanted to find the set of protein regions that consistently preserve their disordered nature. In our view it is better to obtain a relatively restricted but stable set of regions, than getting a larger set that is diluted with false positive cases. Now we repeated the analysis using another prediction method, ESpritz Xray, and we find very similar tendencies as described in the last paragraph of the findings section.

The published threshold value for IUPred that discriminated folded and disordered regions the best is 0.5; this is not a value that we considered to change. In Fig. 1, however, we have also highlighted residues with a prediction score $>0.4$ but $<0.5$ to show that disorder scores often do not drop very steeply and thus the residues surrounding or intervening predicted disordered regions usually receive predicted scores implying high flexibility.
We have now tried to look for even longer regions of 30 consecutive residues. Only 7 ribosomal and 4 nonribosomal proteins retained LDRs of at least 30 consecutive residues in at least three minimal organisms from at least two of the represented bacterial clades. We added two Additional file 1: Tables S3 and S4 to show the results with this parameter, and also describe those in the manuscript.

We did not want to look for regions $<20$ residues because those cannot be considered as LDRs (at least we do not know of any analysis in the ID literature where $<20$ residues long regions were considered as LDRs). 20 residues thus seemed as the ideal choice. The C-terminal region of GroEL, for example, is just between 15 and 25 residues in most organisms regardless of proteome size, which is most probably restricted by the size of the interior cavity. Multiple experiments with deletion mutants for this region demonstrate that true ensemble-like LDRs of around 20 residues can fulfil crucial functions (see literature references [36-39]).

- It could be of interest to analyze some of the protein families with LDR regions in a bit more detail, including sequences from organisms with non-minimal genomes. There might be interesting patterns in the presence/absence of LDRs that are only apparent on a larger data set.

Authors' response: We agree. We extended the analysis, Table 1, all additional tables and the alignments of Fig. 1 with orthologs from non-minimal reference bacteria as explained below.

- In general, the study could benefit from using some "reference organisms" with non-minimal genome from all investigated groups. It can be of interest whether any feature might be associated with being in a minimal genome.

Authors' response: We agree. We extended the analysis, Table 1, all additional tables and the alignments of Fig. 1 with some reference organisms, namely Escherichia coli (strain K12) representing Proteobacteria, Bacteroides vulgatus (strain ATCC 8482) representing Bacteroidetes, and Bacillus subtilis (strain 168) representing the Firmicutes clade. From Tenericutes we did not include a reference proteome because those are best represented by Mycoplasmas, which are included in the dataset anyways. However, we did not accept further protein hits that only show disorder in the reference proteomes because we were explicitly interested in proteins/protein regions that preserve their disordered nature after severe genome minimisation. We only use the reference proteomes to demonstrate that the identified LDRs are also mostly present in those and are thus not a consequence of genome minimization, or the associated fast evolutionary changes and instability of the proteins. 
- The authors might want to comment on whether all the identified LDRs are 'genuine' disordered regions and not coiled coils or other segments commonly predicted to be disordered.

Authors' response: We do not know about the sparsely detected regions within polymerase subunits $b$ and $b$ 'and FtsH, because those are not consistently disordered and not mentioned in the literature as such. However, the other regions that we identified, the C-terminal tails of DnaK, GroEL, a linker within RpoD and the N-terminus of GrpE and InfB, as well as the tail region of SSB were checked and they are all 'genuine' disordered regions, which correspond to missing regions in the respective PDB structures, as now explained in the manuscript.

- It is not described how the authors identified orthologs as gene/protein names might not be conclusive. Kindly comment on this as there are many missing homologs/orthologs indicated in Table 1.

Authors' response: We actually identified orthologs based on gene and protein names, because we found that in case of these minimal organisms and the associated crucial proteins those were conclusive. When absolutely crucial proteins were missing we were also trying to identify those with blast, but we could never find them. For instance we were surprised to see that GroEL was missing in the two Mycoplasmas that are not even among the smallest proteomes, however we could not find any sequence resembling GroEL and finally found publications stating that in many Mycoplasmas the GroEL-GroES system is completely missing (Wong $P$ and Houry WA, 2004). So the missing homologs/orthologs in Table 1 are really missing as a consequence of genome minimization and not due to misannotation.

- The authors might want to comment on the proteins with LDRs found in only one of the investigated proteomes. It can be of interest whether these proteins are in any way associated with being located in a minimal genome.

Authors' response: This is an interesting suggestion but in our view this would be out of the scope of this discovery note. We did not identify anything that seemed specific for minimal genomes, however they have surprisingly many putative genes/proteins regarding their minimalistic nature. Some of those are disordered. Since several works suggest that their proteins need the extensive assistance of chaperones, we were guessing that among those putative proteins there might be disordered chaperones, but we cannot prove this assumption. Also, we are highly restricted with both text length and number of display items, so we would like to stick to the original idea that is, looking for regions that are consistently disordered in different phylogenetic groups after extensive genome reduction.

- Page 5, line 39: "translation initioation factor" is misspelled.
Authors' response: We thank for this remark, we corrected the mistake.

- The two additional xls files could be combined to a single one with the data in two tabs.

Authors' response: We thank for this remark, now all the Additional Tables with IUPred data are arranged as tabs of a single excel file and those with ESpritz are collected in another.

\section{Reviewer 3: Sandor Pongor \\ Reviewer comments:}

The manuscript by Pancsa and Tompa highlights the fact that in organisms with a minimal genome, essential protein functions are linked with structural disorder. The ms is well written and understandable. The figures are clear.

I feel that the message could be made more succinct by emphasizing a few aspects that may not be immediately clear to wider audiences. For instance, i) Why was the set of 13 proteomes selected? Is the finding-i.e. the set of proteins found-sensitive to the selection? The NCBI list of complete (annotated) genomes includes over 80 endosymbionts. In more detail, the Mazumder dataset is optimized for sequence similarity as well bibliographic criteria in the context of all proteomes, while taxonomic coverage within the group of the selected (minimal genome) organisms may be more relevant to this work, at least according to this reviewer. The original paper of Mazumder et al states that CMT55 is superior to other dbase distributions in this respect $\ddot{u}$ can the author explain why they chose the CMT15 dataset?

Authors' response: We thank the reviewer for appreciating our work and his suggestions for improvements. We have chosen the CMT15 dataset because that contains well-annotated species, whose phylogenetic group is assigned. Although this dataset is smaller than the CMT55, the quality of annotations is much better. For Bacteria, the CMT55 dataset is identical to the list of UniProt reference proteomes that currently contains 4159 proteomes (UniProt 08_2016). Back than in 2011 when the Mazumder paper was published they had altogether only 637 proteomes in the CMT55 dataset, while now it is over 5000. The expansion of the sequence space is so fast that annotation procedures can clearly not catch up any more. So, although there are much more proteomes in CMT55, many of those are not well annotated, many come from environmental samples, there are several species represented from the same genus, so it is quite redundant, and there are many unclassified species whose phylogenetic group is not known (for example there are multiple reference proteomes with GW numbers termed as Parcubacteria group bacterium or Microgenomates group bacterium). Also, we have found several mistakes that would potentially affect our dataset, for instance under the 
UniProt code UP000064377 there is a Salmonella enterica subsp. enterica serovar Enteritidis str. LA5 species assigned as a reference proteome with only 102 proteins. It was clear that this annotation cannot be correct, since Salmonella enterica species usually have huge genomes and proteomes with >5000 proteins. We have checked this entry and it turned out that it contains only the proteins of a respective plasmid. Although there are mistakes that are easy to identify and filter out, there are also less shouting annotation mistakes that would not necessarily pop up in the automated data analysis pipeline used here, so we decided to use the smaller but better annotated, more trustworthy CMT15 dataset. For the proteomes that we use, the genes and proteins are also well annotated, so we can rely on the annotated gene and protein names, while for many reference proteomes of the CMT55 dataset they are only numbered with no information on the function of the protein whatsoever. Lastly, if using a considerably larger dataset we could not show the corresponding data table in the manuscript, neither to depict the complete alignments of the orthologs.

ii) Are the orthologues of the identified proteins found in non-reduced genomes also disordered?

Authors' response: For the polymerase subunits we did not find any literature evidence on conserved disordered regions, but for DnaK, DnaJ, GroEL, GrpE, SSB, translation initiation factor 2 (infB) and Peptide chain release factor 1 (prfA) there are analyses in the literature and available protein structures supporting that the respective regions are disordered in orthologs from non-reduced genomes. Now we included three representative reference bacterial proteomes into the analysis to show the disorder status of the identified regions in those and mention the corresponding protein structures.

iii) The identification of disordered proteins relies on the prediction of long disordered stretches. Does the length threshold and the selection of the prediction program influence the findings? Would a different prediction method give different functional predictions?

Authors' response: We have already answered similar questions for Zoltan Gaspari above. The length definitely influences the findings. With a minimum LDR length of 30 consecutive residues, we found less conserved disordered regions, but 7 ribosomal proteins and 4 other proteins still retained LDRs in more than one bacterial clade (see newly added Additional file 1: Table S3 and S4.

Twenty residues seemed as the ideal choice for an $L D R$. We did not want to look for regions $<20$ residues because those cannot be considered as LDRs.

Now we repeated the analysis using another conservative prediction method, ESpritz X-ray, and we find very similar tendencies. Please see the new paragraph before the Discussion section. iii) Can one assign statistical significance to the findings, for instance by simply repeating the predictions with a series of subsets of the selected proteomes?

Authors' response: We could maybe assign statistical significance but we do not think it is necessary. The average fraction of disordered residues in these proteomes ranges between 1 and $12 \%$, with a median of $3.7 \%$ that is extremely low compared to other organisms (Pancsa and Tompa, 2012). The number of identified LDRs was between 1 and 57 in the 13 minimal proteomes including ribosomal proteins, but only 0 to 41 excluding ribosomal proteins (with medians of 17 and 7 regions, respectively). The chance to repeatedly pick the same 20 residues long protein segment just by chance (without assuming the evolutionary conservation of disorder in those regions) is negligibly low. We do not think we must force statistics on that, especially that we are bound by the strict length limitations of the discovery note format. It is clear that most of the regions identified here (except for those in the polymerase subunits and FtsH that appeared in different regions of the orthologous proteins) represent evolutionarily conserved disordered regions that do not only pop up due to prediction or annotation mistakes. Now we show that the respective regions are identified independently from the prediction method used and that they are also mostly disordered in non-minimal reference bacteria from diverse clades.

\section{Additional files}

Additional file 1: Table S1-S4. Ribosomal and non-ribosomal proteins of minimal and reference bacteria with conserved disordered regions of at least 20 or 30 residues predicted by IUPred. (XLS $42 \mathrm{~kb}$ )

Additional file 2: Table S5-S8. Ribosomal and non-ribosomal proteins of minimal and reference bacteria with conserved disordered regions of at least 20 or 30 residues predicted by ESpritz X-ray. (XLS 51 kb)

Funding

The authors receive funding from the Odysseus grant G.0029.12 from Research Foundation Flanders.

Authors' contributions

$\mathrm{RP}$ and PT conceived the analysis. RP performed the analyses and prepared the tables/figures. RP and PT analyzed and discussed the data. RP and PT wrote the manuscript. Both authors read and approved the manuscript.

\section{Competing interests}

The authors declare that they have no competing interests.

Ethical approval and consent to participate

Not applicable.

Received: 25 July 2016 Accepted: 3 September 2016 Published online: 08 September 2016

References

1. Dyson HJ, Wright PE. Intrinsically unstructured proteins and their functions. Nat Rev Mol Cell Biol. 2005;6(3):197-208.

2. Habchi J, Tompa P, Longhi S, Uversky VN. Introducing protein intrinsic disorder. Chem Rev. 2014;114(13):6561-88. 
3. Tompa P. Intrinsically disordered proteins: a 10-year recap. Trends Biochem Sci. 2012;37(12):509-16.

4. van der Lee R, Buljan M, Lang B, Weatheritt RJ, Daughdrill GW, Dunker AK, Fuxreiter M, Gough J, Gsponer J, Jones DT, et al. Classification of intrinsically disordered regions and proteins. Chem Rev. 2014;114(13):6589-631.

5. Wright PE, Dyson HJ. Intrinsically disordered proteins in cellular signalling and regulation. Nat Rev Mol Cell Biol. 2014;16(1):18-29.

6. Dosztanyi Z, Chen J, Dunker AK, Simon I, Tompa P. Disorder and sequence repeats in hub proteins and their implications for network evolution. J Proteome Res. 2006;5(11):2985-95.

7. Dunker AK, Cortese MS, Romero P, lakoucheva LM, Uversky VN. Flexible nets. The roles of intrinsic disorder in protein interaction networks. FEBS J. 2005;272(20):5129-48.

8. Fuxreiter M, Tompa P, Simon I. Local structural disorder imparts plasticity on linear motifs. Bioinformatics. 2007:23(8):950-6.

9. Pancsa R, Fuxreiter M. Interactions via intrinsically disordered regions: what kind of motifs? IUBMB Life. 2012;64(6):513-20.

10. Tompa P, Schad E, Tantos A, Kalmar L. Intrinsically disordered proteins: emerging interaction specialists. Curr Opin Struct Biol. 2015;35:49-59.

11. Varadi M, Zsolyomi F, Guharoy M, Tompa P. Functional advantages of conserved intrinsic disorder in RNA-binding proteins. PLoS One. 2015;10(10):e0139731.

12. Fuxreiter M, Simon I, Bondos S. Dynamic protein-DNA recognition: beyond what can be seen. Trends Biochem Sci. 2011;36(8):415-23.

13. Ward JJ, Sodhi JS, McGuffin LJ, Buxton BF, Jones DT. Prediction and functional analysis of native disorder in proteins from the three kingdoms of life. J Mol Biol. 2004;337(3):635-45.

14. Xue B, Blocquel D, Habchi J, Uversky AV, Kurgan L, Uversky VN, Longhi S. Structural disorder in viral proteins. Chem Rev. 2014;114(13):6880-911.

15. Dunker AK, Obradovic Z, Romero P, Garner EC, Brown CJ. Intrinsic protein disorder in complete genomes. Genome Inform Ser Workshop Genome Inform. 2000;11:161-71.

16. Pancsa R, Tompa P. Structural disorder in eukaryotes. PLoS One. 2012;7(4):e34687

17. Moran NA, Bennett GM. The tiniest tiny genomes. Annu Rev Microbiol. 2014:68:195-215.

18. McCutcheon JP, Moran NA. Functional convergence in reduced genomes of bacterial symbionts spanning 200 My of evolution. Genome Biol Evol. 2010;2:708-18.

19. McCutcheon JP, Moran NA. Extreme genome reduction in symbiotic bacteria. Nat Rev Microbiol. 2012;10(1):13-26.

20. Wang M, Kurland CG, Caetano-Anolles G. Reductive evolution of proteomes and protein structures. Proc Natl Acad Sci U S A. 2011;108(29):11954-8,

21. Wu D, Daugherty SC, Van Aken SE, Pai GH, Watkins KL, Khouri H, Tallon LJ, Zaborsky JM, Dunbar HE, Tran PL, et al. Metabolic complementarity and genomics of the dual bacterial symbiosis of sharpshooters. PLoS Biol. 2006;4(6):e188

22. Chen $C$, Natale DA, Finn RD, Huang $H$, Zhang J, Wu CH, Mazumder $R$. Representative proteomes: a stable, scalable and unbiased proteome set for sequence analysis and functional annotation. PLoS One. 2011;6(4):e18910.

23. Dosztanyi Z, Csizmok V, Tompa P, Simon I. IUPred: web server for the prediction of intrinsically unstructured regions of proteins based on estimated energy content. Bioinformatics. 2005;21(16):3433-4.

24. Dosztanyi Z, Csizmok V, Tompa P, Simon I. The pairwise energy content estimated from amino acid composition discriminates between folded and intrinsically unstructured proteins. J Mol Biol. 2005;347(4):827-39.

25. Lazar T, Schad E, Szabo B, Horvath T, Meszaros A, Tompa P, Tantos A. Intrinsic protein disorder in histone lysine methylation. Biol Direct. 2016;11:30.

26. Potenza E, Di Domenico T, Walsh I, Tosatto SC. MobiDB 2.0: an improved database of intrinsically disordered and mobile proteins. Nucleic Acids Res. 2015;43(Database issue):D315-320.

27. Peng Z, Oldfield CJ, Xue B, Mizianty MJ, Dunker AK, Kurgan L, Uversky VN. A creature with a hundred waggly tails: intrinsically disordered proteins in the ribosome. Cell Mol Life Sci. 2014:71(8):1477-504.

28. Sievers F, Higgins DG. Clustal Omega, accurate alignment of very large numbers of sequences. Methods Mol Biol. 2014;1079:105-16.

29. Berman HM, Westbrook J, Feng Z, Gilliland G, Bhat TN, Weissig H, Shindyalov IN, Bourne PE. The protein data bank. Nucleic Acids Res. 2000;28(1):235-42.
30. Murakami KS. X-ray crystal structure of Escherichia coli RNA polymerase sigma70 holoenzyme. J Biol Chem. 2013;288(13):9126-34.

31. Myasnikov AG, Marzi S, Simonetti A, Giuliodori AM, Gualerzi CO, Yusupova G, Yusupov M, Klaholz BP. Conformational transition of initiation factor 2 from the GTP- to GDP-bound state visualized on the ribosome. Nat Struct Mol Biol. 2005;12(12):1145-9.

32. Simonetti A, Marzi S, Billas IM, Tsai A, Fabbretti A, Myasnikov AG, Roblin P, Vaiana AC, Hazemann I, Eiler D, et al. Involvement of protein IF2 N domain in ribosomal subunit joining revealed from architecture and function of the full-length initiation factor. Proc Natl Acad Sci U S A. 2013;110(39):15656-61.

33. Sprink T, Ramrath DJ, Yamamoto H, Yamamoto K, Loerke J, Ismer J, Hildebrand PW, Scheerer P, Burger J, Mielke T, et al. Structures of ribosomebound initiation factor 2 reveal the mechanism of subunit association. Sci Adv. 2016;2(3):e1501502.

34. Korostelev A, Zhu J, Asahara H, Noller HF. Recognition of the amber UAG stop codon by release factor RF1. EMBO J. 2010;29(15):2577-85.

35. Shin DH, Brandsen J, Jancarik J, Yokota H, Kim R, Kim SH. Structural analyses of peptide release factor 1 from Thermotoga maritima reveal domain flexibility required for its interaction with the ribosome. J Mol Biol. 2004;341(1):227-39.

36. Machida K, Kono-Okada A, Hongo K, Mizobata T, Kawata Y. Hydrophilic residues 526 KNDAAD 531 in the flexible C-terminal region of the chaperonin GroEL are critical for substrate protein folding within the central cavity. J Biol Chem. 2008;283(11):6886-96.

37. Ishino S, Kawata Y, Taguchi H, Kajimura N, Matsuzaki K, Hoshino M. Effects of C-terminal Truncation of Chaperonin GroEL on the Yield of In-cage Folding of the Green Fluorescent Protein. J Biol Chem. 2015;290(24):15042-51.

38. Suzuki M, Ueno T, lizuka R, Miura T, Zako T, Akahori R, Miyake T, Shimamoto $\mathrm{N}$, Aoki M, Tanii T, et al. Effect of the C-terminal truncation on the functional cycle of chaperonin GroEL: implication that the C-terminal region facilitates the transition from the folding-arrested to the folding-competent state. J Biol Chem. 2008;283(35):23931-9.

39. Weaver J, Rye HS. The C-terminal tails of the bacterial chaperonin GroEL stimulate protein folding by directly altering the conformation of a substrate protein. J Biol Chem. 2014;289(33):23219-32.

40. Smock RG, Blackburn ME, Gierasch LM. Conserved, disordered C terminus of DnaK enhances cellular survival upon stress and DnaK in vitro chaperone activity. J Biol Chem. 2011;286(36):31821-9.

41. Assimon VA, Southworth DR, Gestwicki JE. Specific binding of tetratricopeptide repeat proteins to heat shock protein 70 (Hsp70) and heat shock protein 90 (Hsp90) is regulated by affinity and phosphorylation. Biochemistry. 2015;54(48):7120-31.

42. Hohfeld J, Minami Y, Hartl FU. Hip, a novel cochaperone involved in the eukaryotic Hsc70/Hsp40 reaction cycle. Cell. 1995;83(4):589-98.

43. Raghunathan S, Kozlov AG, Lohman TM, Waksman G. Structure of the DNA binding domain of E. coli SSB bound to ssDNA. Nat Struct Biol. 2000;7(8):648-52.

44. Lohman TM, Ferrari ME. Escherichia coli single-stranded DNA-binding protein: multiple DNA-binding modes and cooperativities. Annu Rev Biochem. 1994:63:527-70.

45. Romer R, Schomburg U, Krauss G, Maass G. Escherichia coli single-stranded DNA binding protein is mobile on DNA: 1H NMR study of its interaction with oligo- and polynucleotides. Biochemistry. 1984;23(25):6132-7.

46. Shereda RD, Kozlov AG, Lohman TM, Cox MM, Keck JL. SSB as an organizer/ mobilizer of genome maintenance complexes. Crit Rev Biochem Mol Biol. 2008:43(5):289-318.

47. Savvides SN, Raghunathan S, Futterer K, Kozlov AG, Lohman TM, Waksman $\mathrm{G}$. The C-terminal domain of full-length E. coli SSB is disordered even when bound to DNA. Protein Sci. 2004;13(7):1942-7.

48. Antony E, Weiland E, Yuan Q, Manhart CM, Nguyen B, Kozlov AG, McHenry CS, Lohman TM. Multiple C-terminal tails within a single E. coli SSB homotetramer coordinate DNA replication and repair. J Mol Biol. 2013:425(23):4802-19.

49. Marceau AH. Functions of single-strand DNA-binding proteins in DNA replication, recombination, and repair. Methods Mol Biol. 2012:922:1-21.

50. Lu D, Keck JL. Structural basis of Escherichia coli single-stranded DNAbinding protein stimulation of exonuclease I. Proc Natl Acad Sci U S A. 2008;105(27):9169-74.

51. Kozlov AG, Weiland E, Mittal A, Waldman V, Antony E, Fazio N, Pappu RV, Lohman TM. Intrinsically disordered C-terminal tails of E. coli single-stranded DNA binding protein regulate cooperative binding to single-stranded DNA. J Mol Biol. 2015;427(4):763-74. 
52. Dalbey RE, Wang P, van Dij JM. Membrane proteases in the bacterial protein secretion and quality control pathway. Microbiol Mol Biol Rev. 2012;76(2):311-30.

53. Langklotz S, Baumann U, Narberhaus F. Structure and function of the bacterial AAA protease FtsH. Biochim Biophys Acta. 2012;1823(1):40-8.

54. Sauer RT, Baker TA. AAA+ proteases: ATP-fueled machines of protein destruction. Annu Rev Biochem. 2011;80:587-612.

55. Walsh I, Martin AJ, Di Domenico T, Tosatto SC. ESpritz: accurate and fast prediction of protein disorder. Bioinformatics. 2012;28(4):503-9.

56. Kovacs D, Szabo B, Pancsa R, Tompa P. Intrinsically disordered proteins undergo and assist folding transitions in the proteome. Arch Biochem Biophys. 2013;531(1-2):80-9.

57. Kovacs D, Kalmar E, Torok Z, Tompa P. Chaperone activity of ERD10 and ERD14, two disordered stress-related plant proteins. Plant Physiol. 2008:147(1):381-90.

58. Liu J, Perumal NB, Oldfield CJ, Su EW, Uversky VN, Dunker AK. Intrinsic disorder in transcription factors. Biochemistry. 2006;45(22):6873-88.

\section{Submit your next manuscript to BioMed Central} and we will help you at every step:

- We accept pre-submission inquiries

- Our selector tool helps you to find the most relevant journal

- We provide round the clock customer support

- Convenient online submission

- Thorough peer review

- Inclusion in PubMed and all major indexing services

- Maximum visibility for your research

Submit your manuscript at www.biomedcentral.com/submit 